\title{
A New Visibility Partition for Affine Pattern Matching
}

\author{
Michiel Hagedoorn`, Mark Overmars, and Remco C. Veltkamp \\ Department of Computer Science, Utrecht University \\ Padualaan 14, $3584 \mathrm{CH}$ Utrecht, The Netherlands \\ $\{$ mh, markov, Remco.Veltkamp\}@cs . uu.nl
}

\begin{abstract}
Visibility partitions play an important role in computer vision and pattern matching. This paper studies a new type of visibility, reflection-visibility, with applications in affine pattern matching: it is used in the definition of the reflection metric between two patterns consisting of line segments. This metric is affine invariant, and robust against noise, deformation, blurring, and cracks. We present algorithms that compute the reflection visibility partition in $O((n+k) \log (n)+v)$ randomised time, where $k$ is the number of visibility edges (at most $O\left(n^{2}\right)$ ), and $v$ is the number of vertices in the partition (at most $\left.O\left(n^{2}+k^{2}\right)\right)$. We use this partition to compute the reflection metric in $O\left(r\left(n_{A}+n_{B}\right)\right)$ randomised time, for two line segment unions, with $n_{A}$ and $n_{B}$ line segments, separately, where $r$ is the complexity of the overlay of two reflection-visibility partitions (at most $O\left(n_{A}{ }^{4}+n_{B}{ }^{4}\right)$ ).
\end{abstract}

\section{Introduction}

The visibility from a particular viewpoint in a pattern gives a local description of the pattern. The visibilities from all possible viewpoints give a complete representation of the pattern. This insight led to the use of visibility for pattern matching. Visibility has been used for object-recognition as early as 1982, see Chakravarty and Freeman [6]. Visibility is defined in terms of affine geometry, the concept does not depend on Euclidean distances. Therefore, visibility can be used as a tool for affine invariant shape recognition and affine pattern matching. We use a strong type of visibility to compute an affine invariant pattern metric, called the reflection metric. This metric is affine invariant, and robust against noise, deformation, blurring, and cracks, see Section 2.

A well-studied structure related with visibility is the visibility graph. For a collection of $n$ planar line segments, the visibility graph is the graph having the endpoints of the line segments as vertices, and edges between vertices for which the corresponding endpoints can be connected by an open line segment disjoint with all segments in the collection. A trivial algorithm, consisting of three nested loops, computes the visibility graph in $O\left(n^{3}\right)$ time. Lee [18] was the first to improve this, by giving an $O\left(n^{2} \log (n)\right)$ time algorithm. Optimal $O\left(n^{2}\right)$

\footnotetext{
^ supported by Philips Research
} 
time algorithms were found by Welzl [23], and Asano et al. [3]. If the number of visibility edges is $k$, an algorithm by Pocchiola and Vegter [22] computes the visibility graph in $O(n \log (n)+k)$ time and $O(n)$ space.

A plausible approach to using visibility for pattern matching would be visibility graph recognition, see Ghosh [9], and Everett [8]. However, visibility graph recognition is a computationally expensive problem, see Lin and Skiena [19]. Moreover, visibility graphs depend heavily on the topology of the pattern, and are therefore by themselves not suitable for robust pattern matching.

Our approach uses a special form of visibility, called reflection-visibility, for defining a similarity measure on line patterns. This measure, the reflection metric, is affine invariant by definition. That is, the distance between affine transformed patterns $t(A)$ and $t(B)$ equals the distance between the original $A$ and $B$. The reflection metric is robust. It responds proportionally when lines are deformed a little, slightly translated copies of existing lines are added, small cracks are made in lines, and small new lines are added.

To compute the reflection metric we need to know the structure of the visibility partition corresponding to a special type of visibility. Visibility partitions consists of equivalence classes with constant combinatorial visibility. Plantinga and Dyer [21] call this structure the viewpoint space partition. The dual of the visibility partition is called the aspect graph, see Kriegman and Ponce [17], Bowyer and Dyer [5], and Gigus et al. [11]. The number of possible views, the size of the visibility partition, was investigated, under varying assumptions, by Agarwal and Sharir [1], and de Berg et al. [7]. For polygons, results about visibility partitions were found by Guibas et al. [13], Aronov et al. [2], and Bose et al. [4].

In this paper, we focus on the structure of visibility partitions as two-dimensional arrangements. We will investigate an alternative visibility partition, called reflection-visibility. As a start, we consider the standard visibility partition. We use an alternative way to define and describe it that is useful for the other types of visibility. After that, the analysis becomes more interesting as we proceed to the reflection-visibility partition. Let $k$ be the number of visibility edges (at most quadratic in $n$ ) and $v$ be the number of vertices in the partition (at most quadratic in $n+k)$. We present randomised algorithms that compute the partitions in $O((n+k) \log (n)+v)$ time. We use this to compute the reflection distance. We assume a model of computation in which the absolute value of any rational function, a quotient of polynomials of degree at most $d$, can be integrated over any triangle in $\Theta(d)$ time. Let $A$ and $B$ be unions of $n_{A}$ and $n_{B}$ segments, respectively. If the overlay of the two corresponding reflection-visibility partitions has complexity $r$, the reflection distance between $A$ and $B$ can be computed in $O\left(r\left(n_{A}+n_{B}\right)\right)$ time.

\section{The Reflection Metric}

The reflection metric, introduced in [16,14], defines a distance between finite unions of algebraic curve segments in the plane. If $A$ and $B$ are such unions, the reflection distance is denoted as $d_{\mathrm{R}}(A, B)$. The reflection metric turns the 
patterns $A$ and $B$ into functions $\rho_{A}, \rho_{B}: \mathbb{R}^{2} \rightarrow \mathbb{R}$, after which the integrated absolute difference of these functions is normalised. The functions are defined using a strong form of visibility, called reflection-visibility. We defer the exact definition of the reflection metric until Section 5 .

Figure 1 shows a two-dimensional pattern $A$ consisting of a finite number of straight line segments. Figure 2 shows the corresponding function evaluated on a discrete lattice, represented as a grey-scale image in which black corresponds with value 0 . The example pattern is hieroglyphic 'A1' obtained from the hieroglyphics sign list, see [12].

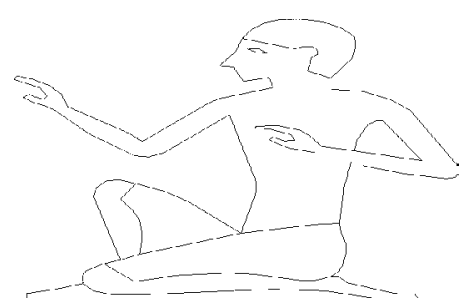

Fig. 1. A straight line pattern

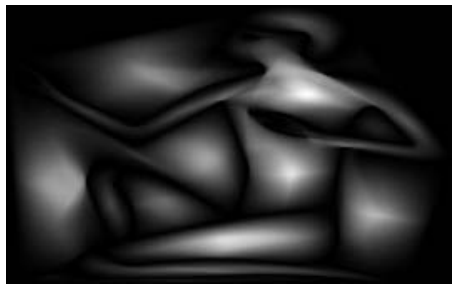

Fig. 2. The function $\rho_{A}$ evaluated on a discrete lattice

Let $T$ be the group of affine transformations on $\mathbb{R}^{2}$. The reflection metric is invariant for $T$, meaning that $d_{\mathrm{R}}(t(A), t(B))=d_{\mathrm{R}}(A, B)$ for any affine transformation $t \in T$. As a result, $d_{\mathrm{R}}$ can be used to construct a metric on affine shapes, patterns modulo affine transformation:

$$
D_{R}(T(A), T(B))=\inf _{t \in T} d_{\mathrm{R}}(t(A), B) .
$$

The reflection metric is robust for various types of effects caused by discretisation and unreliable feature extraction. Slight deformations of patterns only increase or decrease distances slightly. Introducing blur, by adding new lines near existing lines in a pattern, only causes a proportional change in the reflection distance. Making cracks in the interior of lines, splitting them up into multiple smaller ones, only changes the distance proportional to the length of these cracks.

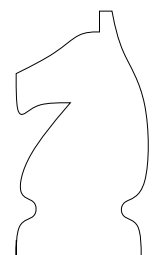

Fig. 3. Deformation, blur, cracks and noise Adding noise in the form of new lines, far away from other lines, only increases the distance proportionally to the length of the added line. Figure 3 illustrates the effects of deformation, blur, cracks, and noise. The left pattern is the "original", the right pattern is "affected" by the four types of distortion.

In Section 5, we show that the reflection metric can be computed by constructing and traversing an arrangement, which is the overlay of two reflectionvisibility partitions. In the following sections, we characterise the visibility and 
reflection visibility partitions. We will give a randomised algorithm that is optimal in the number of segments $n$.

\section{Visibility Partitions}

Let $\mathcal{S}=\left\{S_{1}, \ldots, S_{n}\right\}$ be a collection of closed line segments and let $P=$ $\left\{p_{1}, \ldots, p_{m}\right\}$ be the corresponding set of endpoints. In all that follows, we assume that the endpoints are in general position. For convenience, set $A=\bigcup \mathcal{S}$. We say a point $y \in \mathbb{R}^{2}$ is visible from $x \in \mathbb{R}^{2}$, if the open line segment $\overline{x y}$ is disjoint with $A$. For any viewpoint $x \in \mathbb{R}^{2}$, define the visible part of $A$ as the subset $\mathrm{Vp}_{A}(x) \subseteq A$ given by:

$$
\mathrm{Vp}_{A}(x)=\{a \in A \mid A \cap \overline{x a}=\varnothing\} .
$$

This set is sometimes called the visibility polygon of $x$ in $A$, see [3]. The visibility star $\operatorname{Vst}_{A}(x)$ is the union of all open line segments connecting the viewpoint $x$ with the visible part of $A$ :

$$
\operatorname{Vst}_{A}(x)=\bigcup_{a \in \operatorname{Vp}_{A}(x)} \overline{x a}
$$

Visibility stars are similar to view zones, see [20] pp. 383-391. A view zone is a visibility star extended with all infinite rays from $x$ disjoint with $A$. Figure 4 shows a visibility star for an example consisting of eight line segments with thirteen distinct endpoints. The visible part $\mathrm{Vp}_{A}(x)$ is drawn thick. The visibility star $\operatorname{Vst}_{A}(x)$ is the light grey region, including the dotted lines.

Consider the endpoints and segments bounding the visibility $\operatorname{star} \operatorname{Vst}_{A}(x)$ ordered by slope with respect to $x$. This describes the structure of the visibility star. The visibility star is a finite union of triangles. Each triangle is an intersection of three half-planes. Two of the half-planes are bounded by lines through $x$ and a point in $P$. The third half-plane is bounded by the line through a segment of $\mathcal{S}$. If a segment $S_{i} \in \mathcal{S}$ has a visible endpoint $p_{j}$, and $x$ is collinear with $S_{i}$, then the triangle "degenerates" to the open line segment $\overline{x p_{j}}$. We are interested in the regions in the plane in which the structure of the visibility star is constant.

We simplify the presentation by introducing an additional "line segment". Let $D$ be an open rectangle containing the union of segments $A$, and let $S_{0}$ be its boundary. We will simply call $S_{0}$ a segment. This gives an extended collection of segments $\mathcal{S}^{\prime}=\left\{S_{0}\right\} \cup \mathcal{S}$. Let $A^{\prime}=S_{0} \cup A$. Each ray starting from any point in $D$, intersects $A^{\prime}$.

We need a compact description of the structure of the visibility $\operatorname{star} \operatorname{Vst}_{A}(x)$, for any viewpoint $x \in D$. For this purpose, we define a collection of identifiers, referring either to segments or endpoints. An identifier is an (integer) index subscripted with a $p$ or an $s$, indicating an endpoint or a segment, respectively. We order the identifiers linearly as follows: $0_{s}<1_{s}<\cdots<n_{s}<1_{p}<\cdots<m_{p}$. Each point $a \in A^{\prime}$ is assigned an identifier $\operatorname{id}(a)$ as follows. If $a=p_{i}$, then set $\operatorname{id}(a)=i_{p}$. If $a \in S_{i}-P$, then set $\operatorname{id}(a)=i_{s}$. 


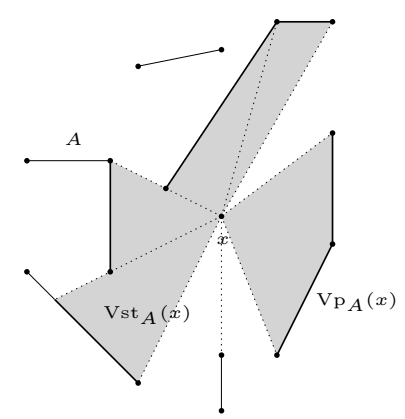

Fig. 4. A visibility star

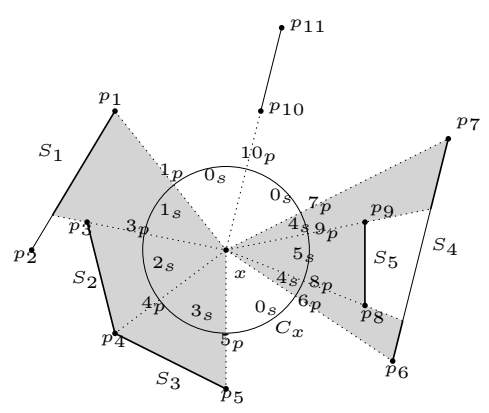

Fig. 5. A view map

We represent the structure of the visibility star by a tuple identifiers. Choose any closed disc centred at $x$ disjoint with $A$. The boundary of such a disc is called the view circle, denoted by $C_{x}$. We give each point $c \in C_{x}$ a label $l(c)$, an identifier, as follows. For each point $a \in A^{\prime}$ visible from $x$, compute the intersection $c \in C_{x} \cap \overline{x a}$ and set $l(c)=\operatorname{id}(a)$.

The view circle $C_{x}$ is a disjoint union of inverse images $l^{-1}(d)$, for each identifier $d$. Each non-empty inverse image $l^{-1}(d)$, the subset of points in $C_{x}$ with label $d$, can be decomposed into its connected components. These components are either single points or open arcs. The view map of $x$, denoted with $\operatorname{Vmp}_{\mathcal{S}}(x)$, is a labeled circuit graph whose vertices are the components with their constant labels. We call vertices labeled with endpoint identifiers $\left(i_{p}\right)$ p-vertices. We call vertices labeled with segment identifiers $\left(i_{s}\right)$ s-vertices. Edges of $\operatorname{Vmp}_{\mathcal{S}}(x)$ are defined by pairs of vertices, constant-label components, with intersecting closures. Figure 5 shows the labeled view circle, inducing the view map, for a collection of four closed line segments having nine distinct endpoints. Labels of $p$-vertices are indicated on the dotted lines on the outside of $C_{x}$. Labels of $s$ vertices are indicated inside the view circle between successive dotted lines.

The view map $\operatorname{Vmp}_{\mathcal{S}}(x)$ can be represented using a tuple of labels encountered when traversing all edges, starting with some initial vertex and some incident edge. Of all possible tuples, the lexicographically smallest one represents the view map. This representation does not depend on the direction (clockwise or counter-clockwise) in which the labels occur on the view circle. We identify the view map with this unique tuple. In the situation of Figure 5 this gives $\operatorname{Vmp}_{\mathcal{S}}(x)=\left(0_{s}, 1_{p}, 1_{s}, 3_{p}, 2_{s}, 4_{p}, 3_{s}, 5_{p}, 0_{s}, 6_{p}, 4_{s}, 8_{p}, 5_{s}, 9_{p}, 4_{s}, 7_{p}, 0_{s}, 10_{p}\right)$.

The view map $\operatorname{Vmp}_{\mathcal{S}}(x)$ is a function of points $x \in D$. Define points $x, y \in D$ to be equivalent if their view maps (labeled graphs) are isomorphic, that is, $\operatorname{Vmp}_{\mathcal{S}}(x)=\operatorname{Vmp}_{\mathcal{S}}(y)$. This equivalence relation results in a partition of $D$ into equivalence classes. This visibility partition is denoted by $\mathcal{Q}_{v}(\mathcal{S})$. If $x$ and $y$ lie

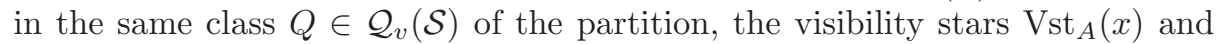
$\operatorname{Vst}_{A}(y)$ have the same structure. 
The visibility partition is affine invariant: the partition for the affine transformed set $\mathcal{S}$ equals the affine transformed partition (including the labels). The reflection-visibility partition also has this property. A single class in the visibility partition can have more than one connected component. In the example of a single segment $\mathcal{S}=\left\{S_{1}\right\}$ with endpoints $P=\left\{p_{1}, p_{2}\right\}$, the open half-planes left and right of $S_{1}$ are the connected components of the equivalence class in $\mathcal{Q}_{v}(\mathcal{S})$ having view map $\left(0_{s}, 1_{p}, 1_{s}, 2_{p}\right)$.

The visibility partition has the structure of an arrangement induced by a finite union of closed line segments. Each cell in this arrangement is a connected component of an equivalence class in the partition. As the viewpoint $x$ moves continuously within $D$, changes occur in $\operatorname{Vmp}_{\mathcal{S}}(x)$. Each time such a change occurs, the set of vertices visible from $x$ changes. The sets of viewpoints $x$ on which changes in the viewmap occur form one-dimensional boundaries in the arrangement describing the visibility partition.

We construct a collection of "event segments" for the view map. Let $E_{\mathcal{S}}$ be the collection of (directed) edges in the visibility graph. That is, $E_{\mathcal{S}}$ consist of all pairs of endpoint-indices $(i, j)$ such that $p_{j}$ is visible from $p_{i}$. We extend $E_{\mathcal{S}}$ to a collection $E_{\mathcal{S}}^{\prime}$ by also including the endpoint-index pairs of each segment in $\mathcal{S}$. Given an endpoint $p_{i}$, sort all endpoints $p_{j_{k}}$, with $\left(i, j_{k}\right) \in E_{\mathcal{S}}^{\prime}$, on clockwise angle. This results in a list of endpoint identifiers $j_{1}, \ldots, j_{c}$. Let $s_{k}$ be the segmentidentifier of the segment visible from $p_{i}$ inbetween the angles of $p_{j_{k}}$ and $p_{j_{k+1}}$ relative to $p_{i}$ (where $k+1$ is modulo $c$ ). We construct event segments bounding the set of points in $D$ from which $p_{i}$ is visible.

First, we define the collection of event segments $\mathcal{P}_{i}$. For each $k=1, \ldots, c$, we include in $\mathcal{P}_{i}$ the closure of the visible part of segment $S_{s_{k}}$ (visible from $p_{i}$ ). This includes parts of the special segment with index $0_{s}$.

Second, we construct a segment collection $\mathcal{B}_{i}$ connecting pairs of segments in $\mathcal{P}_{i}$. For each $k=1, \ldots, c$, we construct a closed segment between the two intersections of ray $\left(p_{i}, p_{j_{k}}\right)$ with segments in $\mathcal{S}^{\prime}$. If these two intersection coincide, we include no segment in $\mathcal{B}_{i}$, for that particular $k$.

The third and last types of segments $\mathcal{X}_{i}$ are extensions of segments in $\mathcal{S}$. Consider each segment $S$ in $\mathcal{S}$ having endpoint $p_{i}$, and having another endpoint $p_{j}$. Include in $\mathcal{X}_{i}$, the closed segment having endpoints $p_{i}$, and the intersection of $\operatorname{ray}\left(p_{i}, p_{j}\right)$ with $\bigcup \mathcal{P}_{i}$ that is closest to $p_{i}$.

The three types of segments result in the arrangement describing the visibility partition. Let $\mathcal{P}, \mathcal{B}$, and $\mathcal{X}$, denote the unions of $\mathcal{P}_{i}, \mathcal{B}_{i}$, and $\mathcal{X}_{i}$, over all $i=$ $1, \ldots, m$, respectively.

Theorem 1. The boundaries in the visibility partition are formed by the event segments: $\bigcup_{Q \in \mathcal{Q}_{v}(\mathcal{S})} \partial Q=\bigcup \mathcal{P} \cup \cup \mathcal{B} \cup \cup \mathcal{X}$.

Figure 6 shows the visibility partition for four line segments having seven distinct endpoints. The union of $\mathcal{P}$ coincides with $A$. The segments of $\mathcal{B}$ are drawn dashed. The segments of $\mathcal{X}$ are drawn coarse dashed. The points where event segments meet are indicated as dots. The rectangle containing the segments is the "segment" $S_{0}$. 
For a derivation of the worst-case complexity of the visibility partition, see [15]. The visibility partition $\mathcal{Q}_{v}(\mathcal{S})$ corresponding to $\mathcal{S}$ can be computed as follows. First, we compute the visibility graph for $\mathcal{S}$, in $O(n \log (n)+k)$ time using algorithms by Ghosh and Mount [10] or Pocchiola and Vegter [22]. Using the visibility graph, the view map can be computed for each endpoint. A simple algorithm discovers the segment visible between successive visibility edges incident to each endpoint by performing $\log (n)$ iterations over the visibility edges. Include the segments of $\mathcal{S}$ as visibility edges. For each (directed) visibility edge $e=(p, q)$, such that no segment adjacent to $q$ is visible to the left of $q$ relative to $p$, store a pointer $\mathrm{r}(e)$ to the visibility edge incident to $q$ that turns right relative to $e$. If a segment adjacent to $q$ is visible to the left of $q$ (relative to $p$ ), we store a pointer to this segment in $\mathrm{r}(e)$. In each iteration we consider all edges $e$ in the visibility graph. If $\mathrm{r}(e)$ is not a segment blocking the view to the left we replace $\mathrm{r}(e)$ by $\mathrm{r}(\mathrm{r}(e))$. Analogous, we maintain pointers $\mathrm{l}(e)$, where the roles of left and right exchange. After $O(\log (k))=O(\log (n))$ iterations, we have found the segments that block the view directly to the left and the right of each directed visibility edge. This takes a total of $O((n+k) \log (n))$ time. Using this information, we can generate the total collection of event segments in $O(n+k)$ time. Let $v$ be the number of intersections in the collection of event segments thus generated. Using randomised incremental construction we construct a trapezoidal decomposition of this collection, see Mulmuley [20] pp. 84-94, in $O((n+k) \log (n)+v)$ time. The arrangement defined by the event segments can be obtained by merging together trapezoids into polygonal cells. Thus, the visibility partition, represented as an arrangement, can be computed using randomised techniques in $O((n+k) \log (n)+v)$. This results in the following theorem.

Theorem 2. The visibility partition of $n$ segments has worst-case complexity $\Theta\left(n^{4}\right)$. Using randomisation, it can be computed in $O((n+k) \log (n)+v)$ time, where $k=\Theta\left(n^{2}\right)$ is the number of visibility edges, and $v=O\left(n^{2}+k^{2}\right)$ is the number of vertices in the arrangement.

\section{Reflection Partitions}

In this section, we consider a stronger notion of visibility, resulting in different stars and partitions. We say that a point $y \in \mathbb{R}^{2}$ is trans-visible from a point $x \in \mathbb{R}^{2}$ if $y$ is visible from $x$ and $\operatorname{both} \operatorname{ray}(x, y)$ and $\operatorname{ray}(y, x)$ intersect $A$. The trans-visible part of $A$ is given by:

$$
\operatorname{Tp}_{A}(x)=\left\{a \in \operatorname{Vp}_{A}(x) \mid A \cap \operatorname{ray}(a, x) \neq \varnothing\right\} .
$$

The trans-visibility star $\operatorname{Tst}_{A}(x)$ is the union of all open line segments between $x$ and the trans-visible part of $A$ :

$$
\operatorname{Tst}_{A}(x)=\bigcup_{a \in \operatorname{Tp}_{A}(x)} \overline{x a} .
$$




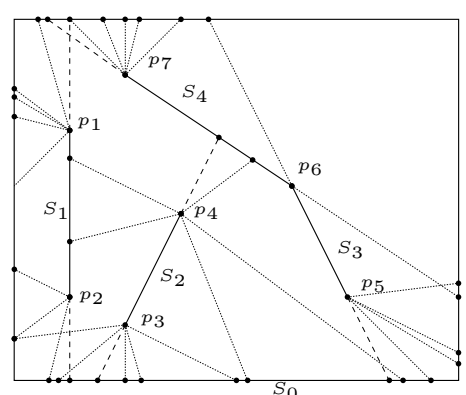

Fig. 6. A visibility partition

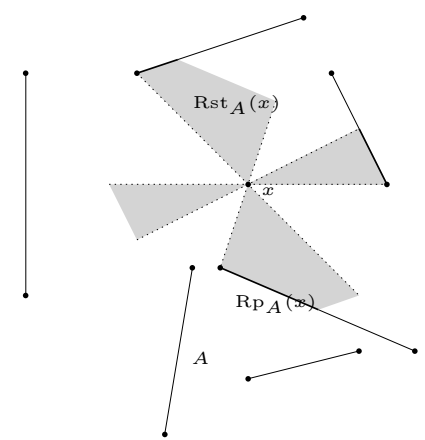

Fig. 7. A reflection star

Based on trans-visibility, we can define a trans-view map, and a trans-visibility partition, see [15].

We say a point $y \in \mathbb{R}^{2}$ is reflection-visible from a point $x$, if $y$ is trans-visible from $x$ and the open segment between $y$ and the reflection of $y$ in $x$ is disjoint with $A$. Observe that in contrast with visibility and trans-visibility, reflectionvisibility is not symmetric. Define the reflection-visible part of $A$ as follows:

$$
\operatorname{Rp}_{A}(x)=\left\{x+v \in \operatorname{Tp}_{A}(x) \mid \overline{(x+v)(x-v)}=\varnothing\right\} .
$$

Define the reflection-visibility star as:

$$
\operatorname{Rst}_{A}(x)=\bigcup\left\{\overline{(x+v)(x-v)} \mid x+v \in \operatorname{Rp}_{A}(x)\right\} .
$$

The reflection-visibility star equals the intersection of a trans-visibility star $\operatorname{Tst}_{A}(x)$ with its reflection around $x$. Figure 7 shows a reflection-visibility star for six disjoint segments. The grey area, including the dotted segments, forms the reflection-visiblity star.

Consider a view circle $C_{x}$ centred at $x$. We label the view circle to describe the structure of the reflection-visibility star at $x$. We define a labeling $l$ of a view circle $C_{x}$, that represents the structure of the reflection visibility star. We use polar coordinates, such that each point $c \in C_{x}$, is represented as $c=(\alpha, r)$, where $r$ is the radius of $C_{x}$. Let $L(x, \alpha)$ be the line through $x$ and $(\alpha, r)$. Let $\epsilon>0$ be smaller than the angle between any two endpoints. There are three cases:

1. If $L(x, \alpha)$ intersects a visible point of $S_{0}$, then $l(c)=0_{s}$.

2. If $L(x, \alpha)$ intersects a visible endpoint $p \in P$ and $L(x, \alpha-\epsilon)$ or $L(x, \alpha+\epsilon)$ intersects a visible point of $S_{0}$, then $l(c)=\operatorname{id}(p)$.

3. In all other cases, set $l(c)=\operatorname{id}(a)$, where $a$ is the visible point in $A \cap L(x, \alpha)$ closest to $x$. 
Overlaps in the rules are resolved by choosing the minimum-index identifier.

The labeling $l$ defines a labeled circuit graph called the reflection view map, denoted by $\operatorname{Rmp}_{\mathcal{S}}(x)$. Figure 8 shows the view circle along with the labels of the reflection-view map. The reflection-visible part is shown thick. The dashed lines are reflections of segments in the view point.

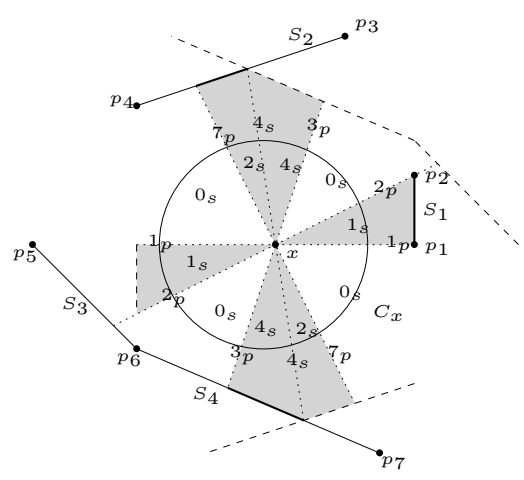

Fig. 8. A reflection-view map

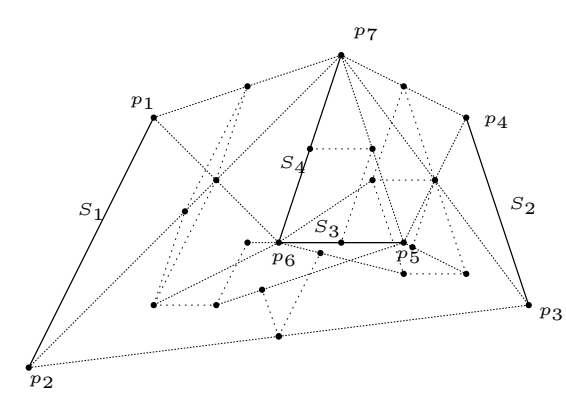

Fig. 9. A reflection-visibility partition

The reflection-view map $\operatorname{Rmp}_{\mathcal{S}}(x)$ at a point $x$ contains the structure of the reflection-visibility star $\operatorname{Rst}_{A}(x)$ at a given point. Starting at some vertex and some initial edge, we obtain a tuple of labels representing the reflection-view map. Since this tuple repeats itself, we only take the first half. We choose the lexicographically smallest half-tuple as a unique representation of $\operatorname{Rmp}_{\mathcal{S}}(x)$. In the situation of Figure 8 this gives $\operatorname{Rmp}_{\mathcal{S}}(x)=\left(0_{s}, 1_{p}, 1_{s}, 2_{p}, 0_{s}, 3_{p}, 4_{s}, 2_{s}\right)$.

We obtain a reflection-visibility partition by identifying points $x, y \in D$ if their reflection-view maps $\operatorname{Rmp}_{\mathcal{S}}(x)$ and $\operatorname{Rmp}_{\mathcal{S}}(y)$ are equal. When moving $x$, if the reflection-view map changes, then the set of reflection-visible endpoints (relative to $x$ ) changes: if we move $x$, either an endpoint identifier, a segment identifier, or an intersection identifier, appears or disappears. In case of an endpoint identifier, the result is immediate. In case of a segment identifier, a change occurs only if one endpoint starts to occlude another one. If an intersection identifier disappears it is replaced by an endpoint identifier. Since a change in the set of endpoints also implies a change in the reflection view map, we can conclude the following. Each class in the reflection-visibility partition is a maximal connected subset of $D$ in which a fixed set of endpoints is reflection-visible.

We construct a collection of event segments for the reflection-view map. Consider an endpoint $p_{i}$. Consider $\mathcal{P}_{i}$ as for visibility. Replace each segment in $\mathcal{P}_{i}$ that lies in $S_{0}$ by a "degenerate" segment consisting of the point $p_{i}$. Now build $\mathcal{B}_{i}$ and $\mathcal{X}_{i}$ using $\mathcal{P}_{i}$ just as in the visibility case.

Consider a scaling transformation $f$ that leaves $p_{i}$ fixed and which has a scaling factor of $1 / 2$. That is, all coordinates relative to $p_{i}$ are multiplied by 
$1 / 2$. To obtain the desired reflection-view map, replace all segments in $\mathcal{P}_{i}, \mathcal{B}_{i}$ and $\mathcal{X}_{i}$ by their images under $f$. Like before, we construct unions of the segment collections over $i=1, \ldots, m$.

Theorem 3. The boundaries in the reflection-visibility partition are formed by the event segments: $\bigcup_{Q \in \mathcal{Q}_{r}(\mathcal{S})} \partial Q=\bigcup \mathcal{P} \cup \cup \mathcal{B} \cup \cup \mathcal{X}$.

Figure 9 shows a reflection-visibility partition. The complexity of the reflection-visibility partition is at most $O\left(n^{4}\right)$. For the $\Omega\left(n^{4}\right)$ worst-case lower bound, see [15]. To compute the reflection-visibility, we can use the same basic techniques to compute the normal visibility partition.

Theorem 4. The reflection-visibility partition of $n$ segments has worst-case complexity $\Theta\left(n^{4}\right)$. Using randomisation, it can be computed in $O((n+k) \log (n)+$ $v$ ) time, where $k=\Theta\left(n^{2}\right)$ is the number of visibility edges, and $v=O\left(n^{2}+k^{2}\right)$ is the number of vertices in the arrangement.

\section{Computing the Reflection Metric}

As an application of the previously defined structures, we use them in computing the reflection metric. Let $\rho_{A}(x)$ be the area of the reflection $\operatorname{star} \operatorname{Rst}_{A}(x)$ for each $x \in \mathbb{R}^{2}$, see Figure 2 for an example. Observe that for points $x$ outside the convex hull of $A$, this area is always zero. If we have two finite unions of line segments $A$ and $B$, the reflection metric $d_{\mathrm{R}}$ is defined as:

$$
d_{\mathrm{R}}(A, B)=\frac{\int_{\mathbb{R}^{2}}\left|\rho_{A}(x)-\rho_{B}(x)\right| d x}{\int_{\mathbb{R}^{2}} \max \left(\rho_{A}(x), \rho_{B}(x)\right) d x} .
$$

The reflection metric can be generalised to finite complexes of $d-1$ dimensional algebraic hyper-surface patches in $d$ dimensions. For this, we refer to [16]. Here, we focus at the computation of the reflection metric for finite unions of segments in the plane.

In Section 2, we emphasised the fact that the reflection metric is robust for deformation, blur, cracks, and noise. This property can be derived from the definition without much difficulty. Given a fixed viewpoint, the change in the area of the visibility star caused by each of the above effects is proportional. The area of the reflection-visibility star, $\rho_{A}(x)$, changes at most twice as much as the area of the visibility star. This pointwise behaviour of the $\rho_{A}$-function is preserved as we integrate it, showing robustness of the reflection metric.

Now, we apply the results from the previous sections to compute the reflection metric. We assume $A$ and $B$ are unions of $n_{A}$ and $n_{B}$ line segments, respectively. having at most $k$ edges in their visibility graphs. The reflection metric can be rewritten as follows:

$$
d_{\mathbb{R}}(A, B)=\frac{2 \int_{\mathbb{R}^{2}}|r(x)| d x}{\int_{\mathbb{R}^{2}}|p(x)| d x+\int_{\mathbb{R}^{2}}|q(x)| d x+\int_{\mathbb{R}^{2}}|r(x)| d x},
$$


where $p(x)=\rho_{A}(x), q(x)=\rho_{B}(x)$, and $r(x)=p(x)-q(x)$. The functions $p, q$ and $r$ are piecewise rational functions in two variables. With piecewise we mean that there is a finite number of triangles covering the support of the function, such that the restriction of the function to each such triangle is a rational function in two variables. The functions $p$ and $q$ are quotients of polynomials of degrees $O\left(n_{A}\right)$ and $O\left(n_{B}\right)$, respectively. The function $r$ is a quotient of polynomials having degree $O\left(n_{A}+n_{B}\right)$. We adopt a model of computation in which the absolute value of a rational function in two variables, can be integrated over a triangular domain in $\Theta(d)$ time, where $d$ is the maximum degree of the polynomial numerator and denominator.

The computation of the integrals of the rational functions $p, q$ and $r$ proceeds as follows. Let $k_{A}$ and $k_{B}$ denote the number of visibility edges corresponding to $A$ and $B$ respectively. First, we compute the visibility graphs of $A$ and $B$, taking times $O\left(n_{A} \log \left(n_{A}\right)+k_{A}\right)$ and $O\left(n_{B} \log \left(n_{B}\right)+k_{B}\right)$, respectively. Using the algorithm sketched in Section 3 , the event segments that correspond to the reflection visibility partition, can be found in $O\left(s_{A} \log \left(n_{A}\right)\right)$ and $O\left(s_{B} \log \left(n_{B}\right)\right)$ time, where $s_{A}=\Omega\left(k_{A}\right)$ and $s_{B}=\Omega\left(k_{B}\right)$ are the number of event segments for $A$ and $B$, respectively. Then, we compute a trapezoidal decomposition for the union of both event segment collections in time $O\left(\left(s_{A}+s_{B}\right) \log \left(n_{A}+n_{B}\right)+v\right)$, where $v$ is the number of intersections. We integrate the absolute values of $p, q$, and $r$ by summing the partial integrals over all trapezoids (each trapezoid is a union of two triangles). In our model of computation, this takes $\Theta\left(n_{A}+n_{B}\right)$ time for each trapezoid. Since the summation of partial integrals dominates the overall complexity, we arrive at the following result.

Theorem 5. Let $A$ and $B$ each be unions of $n_{A}$ and $n_{B}$ segments, respectively. Using randomisation, the reflection distance $d_{\mathrm{R}}(A, B)$ can be computed in $O\left(r\left(n_{A}+n_{B}\right)\right)$ time, where $r$ is the complexity of the overlay of the reflectionvisibility partitions of $A$ and $B$.

\section{Conclusion}

We presented a new metric for pattern matching, the reflection metric. This metric is invariant under the group affine transformations and can therefore be used for affine shape recognition. The reflection metric is robust for patterndefects such as deformation, blur, cracks, and noise. It can be generalised to finite unions of algebraic hyper-surface patches in any dimension.

The reflection metric is defined in terms of reflection visibility. Trans-visibility and reflection-visibility are stronger than visibility. Reflection-visible points are always trans-visible, and trans-visible points are always visible. We analysed the partitions corresponding to the visibilities, starting at normal visibility, and proceeding with reflection-visibility. New types of events emerged, making the resulting partitions more complex.

Constructions show that the worst-case combinatorial complexity for each of the types of partitions is $\Omega\left(n^{4}\right)$. Using randomised incremental construction, each of the corresponding arrangements can be built in $O((n+k) \log (n)+v)$ 
time, where $k$ is the number of visibility edges, and $v$ is the number of intersections in the arrangement. The structure of reflection-visibility partitions can be used to compute the reflection metric for two collections of segments in $O\left(r\left(n_{A}+n_{B}\right)\right)$ randomised time, where $r$ is the complexity of the overlay of two reflection-visibility partitions. Limiting the endpoints of segments to $\mathbb{Z}^{2}$ would not change the complexity of the partitions, since any finite arrangement in $\mathbb{R}^{2}$ can be transformed into an arrangement in $\mathbb{Z}^{2}$. Limiting the segment slopes to set of allowed slopes does not affect the worst-case complexity of the visibility partition, but does affect the complexity of computing the metric, since the maximal polynomial degree of the piecewise function $\rho$ is linear in the number of different segment slopes.

\section{References}

1. P. K. Agarwal and M. Sharir. On the number of views of polyhedral terrains. Discrete \& Computational Geometry, 12:177-182, 1994. 359

2. B. Aronov, L. J. Guibas, M. Teichmann, and L. Zhang. Visibility queries in simple polygons and applications. In ISAAC, pages 357-366, 1998. 359

3. T. Asano, T. Asano, L. Guibas, J. Hershberger, and H. Imai. Visibility of disjoint polygons. Algorithmica, 1:49-63, 1986. 359, 361

4. P. Bose, A. Lubiw, and I. Munro. Efficient visibility queries in simple polygons. In 4th Canadian Conference on Computational Geometry, 1992. To appear in International Journal of Computational Geometry and Applications. 359

5. K. W. Bowyer and C. R. Dyer. Aspect graphs: An introduction and survey of recent results. Int. J. of Imaging Systems and Technology, 2:315-328, 1990. 359

6. I. Chakravarty and H. Freeman. Characteristic views as a basis for threedimensional object recognition. In Proc. SPIE: Robot Vision, pages 37-45, 1982. 358

7. M. de Berg, D. Halperin, M. H. Overmars, and M. van Kreveld. Sparse arrangements and the number of views of polyhedral scenes. Int. J. Computational Geometry \&S Applications, 7(3):175-195, 1997. 359

8. H. Everett. Visibility graph recognition. PhD thesis, Department of Computer Science, University of Toronto, 1990. 359

9. S. K. Ghosh. On recognizing and characterizing visibility graphs of simple polygons. Discrete \& Computational Geometry, 17(2):143-162, 1997. 359

10. S. K. Ghosh and D. M. Mount. An output-sensitive algorithm for computing visibility graphs. SIAM Journal on Computing, 20:888-910, 1991. 364

11. Z. Gigus, J. Canny, and R. Seidel. Efficiently computing and representing aspect graphs of polyhedral objects. IEEE Transactions on Pattern Analysis and Machine Intelligence, 13:542-551, 1991. 359

12. N. Grimal, J. Hallof, and D. van der Plas. Hieroglyphica, sign list. http://www.ccer.theo.uu.nl/hiero/hiero.html, 1993. 360

13. L. J. Guibas, Rajeev Motwani, and Prabhakar Raghavan. The robot localisation problem. SIAM J. Computing, 26(4), 1997. 359

14. M. Hagedoorn. Pattern matching using similarity measures $\mathrm{PhD}$ thesis, Department of Computer Science, Utrecht University, ISBN 90-393-2460-3, 2000. 359

15. M. Hagedoorn, M. H. Overmars, and R. C. Veltkamp. New visibility partitions with applications in affine pattern matching. Technical Report UU-CS-1999-21, 
Utrecht University, Padualaan 14, 3584 CH Utrecht, the Netherlands, July 1999. http://www.cs.uu.nl/docs/research/publication/TechRep.html. 364, 365, 367

16. M. Hagedoorn and R. C. Veltkamp. Measuring resemblance of complex patterns. In L. Perroton G. Bertrand, M. Couprie, editor, Discrete Geometry for Computer Imagery, Lecture Notes in Computer Science 1568, pages 286-298, 1999. Springer. 359,367

17. D. J. Kriegman and J. Ponce. Computing exact aspect graphs of curved objects: Solids of revolution. ACM Symp. Computational Geometry, 5:119-135, 1990. 359

18. D. T. Lee. Proximity and Reachability in the plane. PhD thesis, University of Illinois at Urbana-Champaign, 1978. 358

19. Y. Lin and S. S. Skiena. Complexity aspects of visibility graphs. International Journal of Computational Geometry and Applications, 5:289-312, 1995. 359

20. K. Mulmuley. Computational Geometry: An introduction through randomized algorithms. Prentice Hall, 1994. 361, 364

21. W. H. Plantinga and C. R. Dyer. Visibility, occlusion and the aspect graph. ACM Symp. Computational Geometry, 5(2):137-160, 1990. 359

22. M. Pocchiola and G. Vegter. Topologically sweeping visibility complexes via pseudotriangulations. Discrete \&3 Computational Geometry, 16(4):419-453, 1996. 359, 364

23. E. Welzl. Constructing the visibility graph for n-line segments in $O\left(n^{2}\right)$ time. Inform. Process. Lett., 20:167-171, 1985. 359 